\title{
Improvement of Digital Terrain Model Interpolation Using SFS Techniques with Single Satellite Imagery
}

\author{
Mohammad A. Rajabi, J. A. Rod Blais \\ Dept. of Geomatics Eng., The University of Calgary, 2500, University Dr., NW, \\ Calgary, Alberta, Canada, T2N 1N4 \\ \{marajabi, blais\} @ucalgary.ca
}

\begin{abstract}
The technique of stereo measurements is mainly applied to extract Digital Terrain Model (DTM) height data from stereo images in photogrammetry and remote sensing. Tremendous amounts of local and global DTM data with different specifications are now available. However, there are numerous geoscience and engineering applications which need denser DTM grid data than available. Advanced space technology has provided much single (if not stereo) high-resolution satellite imageries almost worldwide. In cases where only monocular images are available, reconstruction of the object surfaces becomes more difficult. Shape from Shading (SFS) is one of the methods to derive the geometric information about the objects from the analysis of the monocular images. This paper discusses the use of SFS methods with single high resolution satellite imagery to densify regular grids of heights. Three different methodologies are explained and implemented with both simulated and real data. Very encouraging results are obtained and briefly discussed.
\end{abstract}

\section{Introduction}

Digital Terrain Models (DTMs) are simply regular grids of elevation measurements over the land surface. They are used for the analysis of topographical features in GISs and numerous engineering computations. Rajabi and Blais [1] briefly reviewed and referenced a number of sources for DTM and their applications in engineering as well as science.

Stereo measurements from a pair of aerial photographs or satellite images have mainly been used as the primary data in producing DTMs. Information from double or multiple images in overlap areas ensures reliable and stable models for geometric and radiometric processing. Especially recently, with the rapid improvement in remote sensing technology, automated analysis of stereo satellite data has been used to derive DTM data ([2], [3], and [4]).

Today, with the need for the better management of the limited natural resources, there are numerous geoscience and engineering applications which require denser DTM data than available. But due to some reasons such as cloud coverage, technical and/or political limitations, stereo satellite imagery is not available everywhere. 
Obviously, collecting additional height data in the field is a solution, but if not impossible, is either expensive or time consuming or both. While interpolation techniques are fast and cheap, they have their own inherent difficulties and problems, especially in terms of accuracy of interpolation in rough terrain.

On the other hand, the availability of single satellite imagery for nearly all of the Earth is taken for granted nowadays. Unfortunately, reconstruction of objects from monocular images is very difficult, and in some cases, not possible at all. Inverse rendering or the procedure of recovering three-dimensional surfaces of unknown objects from two-dimensional images is an important task in computer vision research. Shape from Shading (SFS) [5] [6], [7] is one of the techniques used for inverse rendering which converts the reflectance characteristics in images to shape information.

This paper discusses the application of SFS techniques to improve the quality of the interpolated DTM grid data with single satellite imagery of better resolution than the DTM data. The idea is highly motivated by the wide availability of satellite remotely sensed imagery such as Landsat TM and SPOT HRV imagery. Section 2 briefly reviews the general SFS problem and the methods implemented in this paper. Section 3 discusses some implementation details of the methods explained in section 2 in more depth. Section 4 provides numerical examples to support the methodology. Last but not least, section 5 ends the paper with some remarks and conclusion.

\section{Shape from Shading}

SFS is one of the methods which transforms single or stereo 2D images to a 3D scene. Basically, it recovers the surface shape from gradual variations of shading in the image. The recovered shape can be expressed either in terrain height $\mathrm{z}(\mathrm{x}, \mathrm{y})$ or surface normal $\vec{N}$ or surface gradient $(\mathrm{p}, \mathrm{q})=(\partial z / \partial x, \partial z / \partial y)$.

Studying the image formation process is the key step to solve the SFS problem. A Lambertian model is the simplest one in which it is assumed that the gray level at each pixel depends only on light source direction and surface normal. Assuming that the surface is illuminated by a distant point source, we have the following equation for the image intensity:

$$
R(x, y)=\rho \vec{N} \cdot \vec{L}=\rho \frac{p l_{1}+q l_{2}+l_{3}}{\sqrt{p^{2}+q^{2}+1}}
$$

where $\rho$ is the surface albedo, $\vec{N}$ is the normal to the surface and $\vec{L}=\left(l_{1}, l_{2}, l_{3}\right)$ is the light source direction. Even with known $\rho$ and $\overrightarrow{\mathrm{L}}$, the SFS problem will still be a challenging subject, as this is one nonlinear equation with two unknowns for each pixel in the image. Therefore, SFS is intrinsically an underdetermined problem and in order to get a unique solution, if there is any at all, we need to have some constraints. 
Based on the conceptual differences in the algorithms, there are three different strategies to solve the SFS problem [1]: 1. Minimization (regularization) approaches 2. Propagation approaches, and 3. Local approaches. A more detailed survey of SFS methods can be found in [8]. The following subsections briefly review the minimization approach, which is widely used in to solve the SFS problem and the other variants of the minimization approach which are used here to enhance the solution.

\subsection{Minimization Approach}

Based on one of the earliest minimization methods, the SFS problem is formulated as a function of surface gradients, while brightness and smoothness constraints are added to ensure that a unique solution exists [10]. The brightness constraint ensures that the reconstructed shape produces the same brightness as the input image. The smoothness constraint in terms of second order surface gradients helps in reconstruction of a smooth surface.

Brooks and Horn [13] defined the error functional:

$$
I=\iint\left\{(E(x, y)-N \cdot L)^{2}+\lambda\left(\left\|N_{x}\right\|^{2}+\left\|N_{y}\right\|^{2}\right)+\mu\left(\|N\|^{2}-1\right)\right\} d x d y
$$

where $\mathrm{E}(\mathrm{x}, \mathrm{y})$ is the gray level in the image, and the constants $\lambda, N_{x}$ and $N_{y}$ are the partial derivatives of the surface normal with respect to $\mathrm{x}$ and $\mathrm{y}$ directions respectively and $\mu$ are Lagrangian multipliers. As it can be seen the functional has three terms: 1) the brightness error which encourages data closeness of the measured images intensity and the reflectance function, 2) the regularizing term which imposes the smoothness on the recovered surface normals, and 3) the normalization constraint on the recovered normals.

The functional is minimized by applying variational calculus and solving the Euler equation: The resulting fixed-point iterative scheme for updating the estimated normal at the location of $(\mathrm{i}, \mathrm{j})$ and epoch $\mathrm{k}+1$, using the previously available estimate from epoch $\mathrm{k}$ is:

$$
N_{i, j}^{k+1}=\frac{1}{1+\mu_{i, j}\left(\varepsilon^{2} / 4 \lambda\right)}\left(\tilde{N_{i, j}^{k}}+\frac{\varepsilon^{2}}{4 \lambda}\left(E_{i, j}-N_{i, j}^{k} \cdot L\right) L\right)
$$

where

$$
\tilde{N}_{i, j}^{k}=\frac{1}{4}\left(N_{i+1, j}^{k}+N_{i-1, j}^{k}+N_{i, j+1}^{k}+N_{i, j-1}^{k}\right) .
$$

There are two comments about this update equation. First, it seems that one has to solve for the Lagrangian multiplier $\mu_{i, j}$ on a pixel-by-pixel basis. However, as it is seen $\mu_{i, j}$ enters the update equation as a multiplying factor which doesn't change the 
direction of the update normal, therefore we can replace that factor by a normalization step. The second comment is about the geometry of the update equation. As it is seen, the update equation is composed of two components. The first one comes from the smoothness constraint while the second one is a response to the physics of image irradiance equation.

The main disadvantage of the Brook and Horn method or any other similar minimization approach is the tendency of over smoothing the solution resulting in the loss of fine detail. Selecting a conservative value for the Lagrangian multiplier is a very challenging issue in this method. However, in an attempt to overcome this problem, Horn [11] starts the solution with a large value for the Lagrangian multiplier and reduces the influence of the smoothness constraint in each iteration as the final solution is approached.

\subsection{Modified Minimization Approaches}

As it was mentioned in the previous section, the update equation is composed of two components, the smoothness part and the data closeness part. As the first attempt to solve the over smoothing problem with the general minimization approach, an adaptive regularization parameter $\lambda(i, j)$ instead of a fixed $\lambda$ is suggested to be used to adaptively control the smoothness over the image space [9]. In each iteration, the space varying regularization parameter at location $(i, j)$ can be determined by the following function:

$$
\lambda_{\text {new }}=\left(1-e^{-\frac{c(i, j)}{V_{T}}}\right) \lambda_{\text {min }}+\left(e^{-\frac{c(i, j)}{V_{T}}}\right) \lambda_{\text {old }}(i, j)
$$

where $c(i, j)$ is the control signal, $V_{T}$ is a time-constant that regulates the rate of exponential decrease and $\lambda_{\min }$ is a preselected minimum value that $\lambda(i, j)$ may have. The control signal is defined as $c(i, j)=\operatorname{abs}\{I(i, j)-R(i, j)\}$, where $\operatorname{abs}\{-\}$ denotes the absolute value and the function $\lambda_{\text {new }}$ is an exponentially decreasing function with the following properties:

$$
\lim _{c(i, j) \rightarrow 0} \lambda_{\text {new }}=\lambda_{\text {old }}(\mathrm{i}, \mathrm{j}) \quad \text { and } \quad \lim _{c(i, j) \rightarrow \infty} \lambda_{\text {new }}=\lambda_{\text {min }}
$$

so that the regularization parameter is only allowed to decrease with the iterations.

Another method to solve the over smoothing problem is to use a robust error kernel in conjunction with curvature consistency instead of a quadratic smoothness. The robust regularizer constraint function can be defined as [14]:

$$
\rho_{\sigma}\left(\left\|N_{x}\right\|\right)+\rho_{\sigma}\left(\left\|N_{y}\right\|\right)
$$

where $\rho_{\sigma}(\eta)$ is a robust kernel defined on the residual $\eta$ and with width parameter $\sigma$. Among different robust kernels, it is proved that the sigmodial-derivative $\mathrm{M}$ - 
estimator, a continuous version of Huber's estimator, has the best properties for handling surface discontinuities [14] and is defined by:

$$
\rho_{\sigma}(\eta)=\frac{\sigma}{\pi} \log \cosh \left(\frac{\pi \eta}{\sigma}\right) .
$$

Applying calculus of variations to the constraint function using the above mentioned kernel results in the corresponding update equation. Here $\sigma$, the width parameter of the robust kernel, is computed based on the variance of the shape index. Based on Koenderink and Van Doorn [12] the shape index is another way of representing curvature information. It is a continuous measure which encodes the same curvature class information as the mean and Gauss curvature, but in an angular representation. In terms of surface normals, the shape index is defined as [12]:

$$
\phi=\frac{2}{\pi} \arctan \left[\frac{\left(N_{x}\right)_{1}+\left(N_{y}\right)_{2}}{\sqrt{\left(\left(N_{x}\right)_{1}-\left(N_{y}\right)_{2}\right)^{2}+4\left(N_{x}\right)_{2}\left(N_{y}\right)_{1}}}\right]
$$

where $(\ldots)_{1}$ and $(\ldots)_{2}$ denote the $\mathrm{x}$ and $\mathrm{y}$ components of the parenthesized vector, respectively. The variance dependence of the kernel is controlled using the exponential function:

$$
\sigma=\sigma_{0} \exp \left(-\left(\frac{1}{N} \sum \frac{\left(\phi_{1}-\phi_{\mathrm{c}}\right)^{2}}{\Delta \phi_{\mathrm{d}}^{2}}\right)^{1 / 2}\right)
$$

where $\sigma_{0}$ is the reference kernel width which we set to one, $\phi_{c}$ is the shape index associated with the central normal of the neighborhood, $\mathrm{N}_{\mathrm{i}, \mathrm{j}}, \phi_{1}$ is one of the neighboring shape index values and $\Delta \phi_{\mathrm{d}}$ is the difference in the shape index between the center values of adjacent curvature classes which is equal to 1/8 [12].

The other modification that can be done on the minimization approach is on the data closeness part of the update equation. We know that the set of surface normals at a point which satisfy the image irradiance equation define a cone about the light source direction. In other words, the individual surface normals can only assume directions that fall on this cone. At each iteration the updated normal is free to move away from the cone under the action of the local smoothness. However, we can subsequently map it back onto the closest normal residing on the cone. This has not only numerical stability advantage but also all normal vectors in the intermediate states are all solutions of image irradiance equation. In other words, the update equation for the surface normals can be written as:

$$
N_{i, j}^{k+1}=\Theta \tilde{N_{i, j}^{k}}
$$


where $\tilde{N_{i, j}^{k}}$ is the surface normal that minimizes the smoothness constraint while $\Theta$ is the rotation matrix which maps the updated normal to the closest normal lying on the cone of ambiguity. The axis of rotation is found by taking the cross-product of the intermediate update with the light source direction:

$$
\theta=-\cos ^{-1}\left(\frac{\tilde{N_{i, j}^{k}} \cdot L}{\left\|\tilde{N_{i, j}^{k} \|}\right\| L \|}\right)+\cos ^{-1} E
$$

\section{Implementation Details}

The main goal of this investigation is to improve the accuracy of the interpolated DTM grid data by applying SFS techniques with the corresponding single satellite imagery, while the original DTM data are used as boundary constraints in the SFS problem.

The basic assumption here is that the satellite imagery has one dyadic order better resolution than the original DTM data. We also assume that 1) the surface is Lambertian (which is questionable in reality), 2) the surface albedo is known (by applying classification techniques to multispectral satellite imageries), 3) the surface is illuminated by a distant point source (sun), and finally 4) the position of the light source is known.

Our approach deals with a patch at a time (see Fig. 2) with forty nine points. Sixteen grid points have known heights (dark circles) and the other thirty three are points with the interpolated heights (unmarked grid points). Our main objective is to improve the accuracy of the interpolation for the five innermost unknown points. The idea of using a patch at a time for parallel processing techniques appears highly attractive for extensive applications

The method essentially consists of three stages: 1) preprocessing, 2) processing, and 3) postprocessing. The preprocessing stage itself has two steps. In the first step, using interpolation (bilinear) techniques, the heights of the unknown points in the patch are estimated. When dealing with the real height data, if there is a gap in height measurements due to any reason (such as existing of rivers or lakes), the whole patch is left untouched.

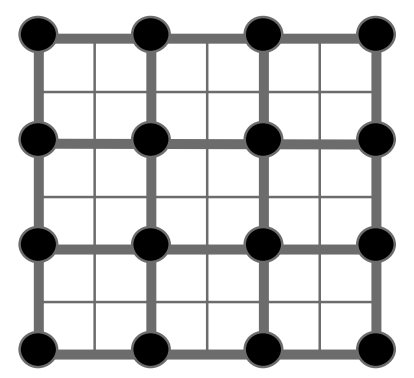

Fig. 1. A patch: Circles are the grid points with known heights and the unmarked ones are the points with the interpolated heights. 
In the second step, using the known grid points, the relative orientation of the inner most square in the patch with respect to the light source is estimated. If this relative orientation implies that the patch is in the shadow, then there would be no useful shading information to improve the accuracy of the interpolated heights. Therefore, in this case the interpolated heights are considered the final height values.

Otherwise, the processing stage for each patch consists of three steps. In the first step, the smoothed surface normals (based on one of the three methods explained in Section (2) are computed. Then in the second step these surface normals are mapped onto the corresponding ambiguity cone. Finally in the third step, the surface normals are passed to an overdetermined (74 equations and 33 unknowns) linear adjustment process to solve for the heights. This is simply done by approximating $\mathrm{p}$ and $\mathrm{q}$ with finite differences in terms of heights. The control goes back to the first step of this stage unless the average difference between the calculated and original image gray values of all the pixels in the patch is less than a predetermined threshold.

The last stage, postprocessing, consists of taking arithmetic means of two solutions for the unknown heights located on the boundary of the innermost square in each patches coming from the neighboring patches, except for the outsides of the peripheral patches.

\section{Numerical Examples}

The methodologies described in Sections 2 and 3 have been tested using a number of numerical examples. One synthetic object with its synthetic imagery and one real DTM data and its corresponding satellite imagery were used in these experiments. With the synthetic object the orientation of the light source was considered as a variable to investigate the effects of relative positions of the object and the light source on the solution.

The synthetic object under investigation is a 1024 by 1024 pixel convex hemisphere with a radius of 250 units, sampled at each 0.5 unit. The corresponding DTM (one dyadic order less than the object, i.e., 512 by 512 pixels) was extracted out from the object. Meanwhile, the corresponding image of the object was created using a Lambertian reflectance model with a much (5 times) denser version of the object. The resulting image was passed through a smoothing filter to get an image with the same density as the original object under study.

The differences between the original object, the Interpolated Grid Solution (IGS) and the SFS solutions were analyzed. Table 1 summarizes these results. SFS1, SFS2, and SFS3 in the table correspond to the three different SFS solutions mentioned in Section 2 respectively. The total number of the patches in this experiment is 215296 . The statistics shown in this table are computed with those pixels which our SFS method was able to update their height values. The other patches are either those for which there is no shading information or the SFS techniques failed to find a solution. In Table 1 nothing is mentioned about the azimuth of the light source. The symmetry of the synthetic object under investigation makes the process independent of the light source azimuth. As it can be seen from this table, SFS techniques have improved the standard deviation of the differences by an average rate of $43.1 \%$. 
Table 1. The convex hemisphere

\begin{tabular}{|c|c|c|c|c|c|c|c|c|}
\hline \multirow{2}{*}{ Elev. } & \multicolumn{2}{|l|}{ Object -IGS } & \multicolumn{2}{l|}{ pbject -SFS1 } & \multicolumn{2}{l|}{ Object-SFS2 } & \multicolumn{2}{l|}{ Pbject -SFS3 } \\
\cline { 2 - 9 } & Mean & Std & Mean & Std & Mean & Std & Mean & Std \\
\hline $30^{\circ}$ & -0.04 & 0.32 & -0.05 & 0.19 & -0.04 & 0.18 & -0.04 & 0.18 \\
\hline $45^{\circ}$ & -0.01 & 0.29 & -0.03 & 0.17 & -0.02 & 0.17 & -0.03 & 0.16 \\
\hline $60^{\circ}$ & 0.02 & 0.32 & 0.03 & 0.18 & 0.03 & 0.17 & 0.02 & 0.17 \\
\hline $\begin{array}{c}\text { Patches } \\
\text { not } \\
\text { updated }\end{array}$ & - & 32294 & 17223 & 10764 \\
\hline
\end{tabular}

The second test object is a real terrain data set from southern Alberta (Waterton), Canada with 25 metre spacing in UTM coordinate system. The original measured DTM data consists of 100 metre spacing grid in addition to the feature points. These measurements were used to interpolate the 25-metre grids. A 1024 by 1024 grid with more than 1300 metre height difference which was extracted out from the four quadrants of NTS 82H04 DTM data file.

The corresponding satellite imagery is a 3 channel SPOT data file with $20 \mathrm{~m}$ resolution which was originally georeferenced to an extended UTM coordinate system. By extracting the coordinates of the distinguished terrain features from the corresponding 1/20000 topographic map sheets, the SPOT imagery was georeferenced to the same coordinate system as the DTM data are. For this purpose 23 points with good distribution in the area under investigation were used. A second order polynomial was used for the purpose of georeferencing. The RMS of georeferencing in $\mathrm{x}$ and $\mathrm{y}$ directions were $2.96 \mathrm{~m}$ and $1.40 \mathrm{~m}$ respectively.

Using PCI software, a principal component transformation was applied and the first channel with $88.52 \%$ energy was selected for these experiments. Finally, the satellite imagery pixel size was changed from $20 \mathrm{~m}$ to $25 \mathrm{~m}$ using a bilinear interpolation method.

To test the efficiency of the SFS methods with the real data set, we tried to reconstruct the $25 \mathrm{~m}$ DTM from $50 \mathrm{~m}$ DTM and $25 \mathrm{~m}$ SPOT imagery. Similar to the simulated data the total number of the patches in this experiment is 215296. The statistics shown in this table are computed with those pixels which our SFS method was able to update their height values. The other patches are either those for which there is no shading information or the SFS techniques failed to find a solution. Moreover, there is no update in height values for those patches which contain natural features such as rivers or lakes where there is no measured height data. Table 2 summarizes the results of our experiment with this data set. As it can be seen from this table, the rate improvement in standard deviation can reach up to $48 \%$. 
Table 2. The real terrain data set

\begin{tabular}{|c|c|c|c|c|c|c|c|c|}
\hline \multicolumn{2}{|c|}{ Object -IGS } & \multicolumn{2}{|c|}{ Object -SFS1 } & \multicolumn{2}{|c|}{ Object-SFS2 } & \multicolumn{2}{|c|}{ Object -SFS3 } & \\
\hline $\begin{array}{c}\text { Mean } \\
(\mathrm{m})\end{array}$ & $\begin{array}{l}\text { Std } \\
(\mathrm{m})\end{array}$ & $\begin{array}{c}\text { Mean } \\
(\mathrm{m})\end{array}$ & $\begin{array}{l}\text { Std } \\
(\mathrm{m})\end{array}$ & $\begin{array}{c}\text { Mean } \\
(\mathrm{m})\end{array}$ & $\begin{array}{l}\text { Std } \\
(\mathrm{m})\end{array}$ & $\begin{array}{c}\text { Mean } \\
(\mathrm{m})\end{array}$ & $\begin{array}{l}\text { Std } \\
(\mathrm{m})\end{array}$ & \\
\hline 0.03 & 14.95 & -0.04 & 10.17 & 0.03 & 8.82 & 0.04 & 7.77 & \\
\hline \multicolumn{2}{|c|}{ - } & \multicolumn{2}{|c|}{$32 \%$} & \multicolumn{2}{|c|}{$41 \%$} & \multicolumn{2}{|c|}{$48 \%$} & $\begin{array}{l}\text { Improvement } \\
\text { of Std }\end{array}$ \\
\hline \multicolumn{2}{|c|}{-} & \multicolumn{2}{|c|}{25835} & \multicolumn{2}{|c|}{2066} & \multicolumn{2}{|c|}{15716} & $\begin{array}{l}\text { Patches not } \\
\text { updated }\end{array}$ \\
\hline
\end{tabular}

\section{Remarks and Conclusions}

SFS is intrinsically an underdetermined problem and in order to get a unique solution one has to implement some kind of constraint(s). Horn's method uses a single Lagrangian multiplier in the formulation of the constraint in the SFS problem. Selecting a conservative value for the Lagrangian multiplier is a very challenging issue in this method. Moreover, Horn's method and other similar approaches have the tendency of over smoothing the solution which results in the loss of fine detail. Obviously, selecting different Lagrangian multipliers for each pixel or cluster of pixels based on the roughness of the surface under study is the solution for this problem.

On the other hand, it seems that using the idea of mapping the computed smoothed normals in each iteration back onto the ambiguity cone as data closeness constraint works very well. This has not only numerical stability advantages but also all normal vectors in the intermediate states are all solutions of image irradiance equation.

Numerical examples show very encouraging results. The fact that the synthetic object is a much smoother surface than the real data set convinces us that the SFS methods should have given better results but this is not the case. The only apparent explanation is the way the synthetic image was constructed. It shows that our method of constructing the synthetic image does not resemble the real life case.

Satellite imagery used in this experiment has a very good quality without any with cloud or snow cover or any other major problem. However, to prove the efficiency of the above mentioned SFS methods, one should test them with different satellite imageries with different quality, resolution, sun position, spectral bands and last but not least different types of terrain.

Fine tuning different variable parameters in the SFS methods, applying classification techniques for having a more realistic value for the albedo, and using a more sophisticated reflectance model are obviously things that should be taken into consideration in future research and development. 


\section{References}

1. Rajabi, M. A., Blais, J. A. R.: Densification of Digital Terrain Model Using Shape From Shading with Single Satellite Imagery. Lecture Notes in Computer Science, Vol. 2074, Springer (2001) 3-12

2. Gugan, D. J., Dowman, I. J.: Topographic Mapping from Spot Imagery. Photgrammetric Engineering and Remote Sensing 54(10) (1988):1409-1414

3. Simard, R., Rochon, G., Leclerc, A.: Mapping with SPOT Imagery and Integrated Data Sets. Invited paper presented at the $16^{\text {th }}$ congress of the International Society for Photogrammetry and Remote Sensing held July 1988 in Kyoto, Japan

4. Tam, A. P.: Terrain Information Extraction from Digital SPOT Satellite Imagery. Dept. of Geomatics Eng., The University of Calgary (1990)

5. Horn, B. K. P.: Shape from Shading: A method for Obtaining the Shape of a Smooth Opaque from One View. Ph.D. Thesis, Massachusetts Ins. of Technology (1970)

6. Horn, B. K. P.: Height and gradient from shading. Int. J. Comput. Vision, 37-5 (1990)

7. Zhang, R., Tsai, P. S., Cryer, J. E., Shah, M.: Analysis of Shape from Shading Techniques. Proc. Computer Vision Pattern Recognition (1994): 377-384

8. Zhang, R., Tsai, P. S., Cryer, J. E., Shah, M.: Shape from Shading: A Survey. IEEE Transaction on Pattern Analysis and Machine Intelligence, Vol. 21, No. 8, August (1999): 690-706

9. Gultekin, A., Gokmen, M.: Adaptive Shape From Shading. ISCIS XI The Eleventh International Symposium on Computer and Information Sciences (1996): 83-92

10. Ikeuchi, K., Horn, B. K. P.: Numerical Shape from Shading and Occluding Boundaries. Artificial Intelligence, Vol. 17, Nos. 1-3 (1981): 141-184

11. Horn, B.K.P.: Height and Gradient from Shading. Int. J. Comput. Vision, Vol. 5, No. 1, (1990): 37-75

12. Koenderink, J. J., van Doorn, A.: Surface Shape and Curvature Scales. Image and Vision Computing, Vol. 10, No. 8, October (1992): 557-565

13. Brooks, M. J., Horn, B. K. P.: Shape and Source from Shading. International Joint Conference on Artificial Intelligence, (1985): 932-936

14. Worthington, P. L., Hancock, E. R.: Needle Map Recovery Using Robust Regularizers. Image and Vision Computing, Vol. 17, (1999): 545-557 